\title{
Explorando el potencial de los dispositivos electrónicos y de las redes sociales en el proceso enseñanza- aprendizaje de los universitarios
}

\author{
EXPLORING THE POTENTIAL OF ELECTRONIC DEVICES AND SOCIAL \\ NETWORKS IN THE TEACHING-LEARNING PROCESS OF UNIVERSITY \\ STUDENTS
}

Amaia Altuzarra Artola; amaia.altuzarra@ehu.eus

Catalina Galvez; catalina.galvez@ehu.eus

Ana Gonzalez; ana.gonzalezflores@ehu.eus

Universidad del País Vasco (UPV/EHU)

\section{RESUMEN}

Este trabajo examina, desde la perspectiva del alumnado, la relación entre diversos métodos de enseñanza y el uso de dispositivos electrónicos y redes sociales en la Facultad de Economía y Empresa de la Universidad del País Vasco (UPV/EHU). La metodología utilizada incluye técnicas estadísticas multivariantes y análisis clúster. Los resultados muestran que la penetración de la tecnología móvil y de las redes sociales en el entorno universitario se caracteriza por una amplia diversidad, tanto en el uso de redes sociales como en la percepción que el alumnado tiene sobre la contribución de las herramientas de la web 2.0 en su proceso de aprendizaje.

Palabras clave: análisis clúster, análisis multivariante, dispositivos electrónicos, redes sociales, Facultad de Economía y Empresa, Universidad del País Vasco

\section{ABSTRACT}

This study examines, from the perspective of the students, the relationship between different teaching methods and the use of electronic devices and social networks in the Faculty of Economics and Business. The methodology includes multivariate statistical techniques and cluster analysis. The results show that the penetration of mobile technology and social networks in the university environment that we have studied is characterized by a wide diversity, because of both the different use of social networks by students and the different perception of the contribution of web 2.0 tools to their learning process.

Keywords: cluster analysis, multivariate analysis, electronic devices, social networks, Faculty of Economics and Business, University of the Basque Country 


\section{INTRODUCCIÓN}

La expansión del uso de internet en las últimas décadas ha generado un mundo más conectado, afectando y transformando los diferentes ámbitos de la vida social y profesional. Entre las principales actividades de los usuarios de internet se encuentra el acceso a las redes sociales, que se han convertido en un espacio de socialización.

El estudio realizado por IAB Spain (Interactive Advertasing Bureau) sobre el uso de las redes sociales en España en 2017, indica que el 82\% de la población española de 16 a 65 años es usuaria de internet y, entre éstos, la penetración de las redes sociales es del $86 \%$. La principal red social es Facebook, empleada por el $91 \%$ de los usuarios. Le siguen WhatsApp (89\%), You Tube (71\%), Twitter (50\%) e Instagram (45\%). El uso principal de las redes sociales es el "social" (chatear/mensajes, ver que hacen los contactos). Pero también es importante para la adquisición de conocimientos y para fines profesionales o de estudios. Por otro lado, según el estudio Navegantes en la Red 2017 realizado por la Asociación de Investigación de Medios de Comunicación (AIMC), el principal dispositivo de acceso a internet es el teléfono móvil, a través del que se conectan el $92 \%$ de los internautas, superando ampliamente al ordenador $72,5 \%$ y a la Tablet (55\%).

Por lo que se refiere al uso que hacen los jóvenes de internet, los datos del Observatorio del Instituto de la Juventud-INJUVE relativos a 2012 muestran que la actividad principal de la población de 15 a 29 años es la "relacional y búsqueda", fundamentalmente definida por la búsqueda de información o documentación (82\%), el uso de las redes sociales (80\%) y el uso del correo electrónico (76\%). Por tanto, internet y las redes sociales se configuran como los escenarios en los que gestionan todas sus relaciones vitales (de amistad, de ocio...) (Bernal y Angulo, 2012; Hernández y Alcoceba, 2015). Así pues, las redes sociales han reconfigurado los campos de actuación y de relación juveniles, tanto en sus dimensiones productivas (tareas escolares, formación, etc.), como de ocio y de relaciones familiares y personales (Contreras y Alvarez, 2017).

Las nuevas formas de comunicación han abierto un espacio para el desarrollo de innovaciones en la educación usando tecnologías basadas en la Web 2.0, que entre sus cualidades incluye la de facilitar la elaboración e intercambio de contenidos generados por los usuarios (Kaplan y Haenlein, 2010). El desarrollo de aplicaciones para dispositivos móviles ha generado, asimismo, extraordinarios cambios tanto en la sociedad como en la educación (El-Hussein y Cronje, 2010). Estas tecnologías permiten a los estudiantes participar de manera activa en el proceso de aprendizaje (Contreras y Álvarez, 2017). Además, ofrecen flexibilidad en cuanto a tiempo, espacio y lugar, favoreciendo lo que se denomina Entonos Personales de Aprendizaje (EPA), entendidos como estrategias para promover el aprendizaje autónomo del estudiante, (Cataldi et al., 2014; Prendes et al., 2016; Humanante-Ramos et al., 2017). Este nuevo paradigma supone una forma interactiva de enseñanza-aprendizaje que requiere la combinación de los métodos tradicionales de enseñanza (clases magistrales, fundamentalmente) con la inclusión de materiales de estudio en plataformas virtuales de aprendizaje y la interacción entre el profesorado y los estudiantes en los entornos web.

Así pues, el dominio de las nuevas tecnologías y de las redes sociales por parte de la denominada "generación Z" favorece el uso de las mismas en el entorno académico universitario (Ebner y Maurer, 2009; Grosseck y Holotescu, 2008; Ebner et al., 2010; Ramsden, 2009; Serra y Martorell, 2017). Estas nuevas tecnologías están transformando la manera en que los estudiantes se comunican, colaboran y aprenden (Tess, 2013). Son varios los trabajos que 
señalan los efectos positivos de estas herramientas en el proceso de enseñanza-aprendizaje. Algunos autores indican que estas tecnologías son vistas por muchos profesores como un mecanismo que les permite transmitir y potenciar sus enseñanzas, así como promover el aprendizaje activo y autónomo de los estudiantes (Selwyn, 2010). Otros consideran que puede ser una herramienta para que los estudiantes se involucren más en el aprendizaje de la materia (Sadaf et al., 2012). Asimismo, algunos trabajos que han examinado la contribución del uso de determinadas redes sociales como Twitter y Facebook a los procesos de aprendizaje han encontrado que el uso de estas aplicaciones favorece el aprendizaje (Dunlap y Lowenthal, 2009; Ophus y Abbitt, 2009; Gao et al., 2012). Sin embargo, otros autores argumentan que hay que ser cautos a la hora de utilizar estas tecnologías en la enseñanza. En este sentido apuntan que los beneficios de estas tecnologías en los resultados de aprendizaje no son concluyentes (Grosch el al., 2014). Otros trabajos señalan que el uso de metodologías basadas en la web 2.0 no mejoran automáticamente los resultados del aprendizaje (Russell, 2001). Friesen y Lowe (2011), por su parte, cuestionan la posibilidad de que las redes sociales fomenten el análisis crítico y el debate, un componente principal del aprendizaje. Sostienen que las redes sociales no fueron desarrolladas para utilizarse en una educación formal (tradicional). Finalmente, otros estudios han constatado que la mayoría de los estudiantes posee una cuenta en las redes sociales, y hace uso de las mismas en su vida personal, pero raramente las utilizan con propósitos educativos (Jones et al., 2010).

En el primer Informe Horizon1 publicado en el año 2005 Johnson, Levine y Smith opinaron que las redes sociales escondían un gran potencial para mejorar la educación. Unos años más tarde, en el Informe Horizon de 2009, los mismos autores destacaron el auge que estaba teniendo el uso de dispositivos móviles subrayando que los individuos organizaban la mayor parte de su información y de sus relaciones sociales en torno a estos dispositivos (Johnson, Levine y Smith, 2009). En los Informes Horizon publicados más recientemente (Johnson, Levine y Smith, 2014; 2015) se ha abundado en estas cuestiones y se ha señalado la conveniencia de encontrar fórmulas que permitan, mediante el uso de estas tecnologías, mejorar el proceso de enseñanza-aprendizaje.

Desde hace algunos años, la mayoría de las instituciones universitarias españolas cuentan con algún tipo de Sistema de Gestión del Aprendizaje (Learning Management System) como por ejemplo Moodle que ofrecen contenidos, facilitan la interacción entre el profesorado y el alumnado, y fomentan la colaboración entre los participantes. Estas estructuras y su uso se han ido extendiendo entre los miembros de la comunidad educativa, y se ha comenzado a explorar el potencial que pueden tener otras herramientas basadas en la web 2.0 en los procesos de enseñanza-aprendizaje. Entre estas herramientas destaca el interés por conocer el papel que las redes sociales pueden jugar en la mejora de la docencia universitaria (Schroeder, Minocha y Schneidert, 2010; Sanchez-Rodriguez et al. 2015; Prendes et al., 2016).

Por tanto, en términos generales podemos concluir que existe cierto consenso de que, en el ámbito educativo, la competencia digital se ha convertido en una competencia básica. En un mundo cada vez más complejo, donde el volumen de información es cada vez mayor, es necesario que los estudiantes tengan la formación que les permita gestionar dicha información utilizando las herramientas tecnológicas que surgen de forma continuada, así como la habilidad de comunicación en entornos virtuales (Revuelta, 2011; Gisbert et al., 2012). Pero, al mismo tiempo, los profesores deberán formarse para adquirir conocimientos que les permita

\footnotetext{
${ }^{1}$ El Proyecto Horizon nació en el año 2002 para tratar de identificar las tendencias tecnológicas actuales y futuras que pueden contribuir a mejorar los procesos de enseñanza-aprendizaje.
} 
aplicar eficazmente estos nuevos dispositivos y tecnologías en el proceso de enseñanza (Ramos et al., 2010).

Este trabajo ${ }^{2}$ examina el uso de los dispositivos móviles (Smartphone y Tablet) y redes sociales (Facebook, Twitter y WhatsApp) entre los estudiantes de la Facultad de Economía y Empresa de la Universidad del País Vasco (UPV/EHU), analizando su opinión sobre la contribución que dichas herramientas, en combinación con los métodos tradicionales de enseñanza, tienen en el proceso y resultado de su aprendizaje. Este análisis permite, en primer lugar, conocer el grado de penetración de las tecnologías móviles y de las redes sociales en el entorno universitario. En segundo lugar, este diagnóstico ayudará el diseño de medidas activas orientadas a un uso más eficiente de las redes sociales en los procesos de enseñanzaaprendizaje.

El resto del trabajo se estructura de la siguiente forma: en la sección segunda se explican los datos utilizados, la sección tercera expone la metodología, en la sección cuarta se presentan e interpretan los resultados obtenidos y, en la última sección se incluye una discusión y las principales conclusiones.

\section{DATOS}

\subsection{Población y tamaño de la muestra}

Para realizar este trabajo, un equipo de profesores de la Facultad de Economía y Empresa de la Universidad del País Vasco (UPV/EHU) diseñamos una encuesta dirigida a los estudiantes de primer curso matriculados en alguno de los grados ofertados por la citada Facultad (Economía, Administración y Dirección de Empresas, Fiscalidad y Administración Pública, Marketing, Finanzas y Seguros y Doble grado en Derecho y Administración y Dirección de Empresas). Se seleccionó a los estudiantes de este curso porque nos permite hacer un seguimiento de la evolución de sus percepciones en los próximos cursos. La población objetivo, por tanto, está formada por los 900 estudiantes de primer curso matriculados en el curso 2015-2016. El cuestionario se depositó en la plataforma Moodle y la cumplimentación del mismo se realizó de forma voluntaria durante el segundo cuatrimestre.

El cuestionario contenía 24 preguntas, 7 de las cuales se referían a la percepción que tenían los alumnos sobre la contribución de los métodos tradicionales de enseñanza-aprendizaje (clases magistrales, clases prácticas, trabajos individuales y/o grupales dentro o fuera del aula) a su proceso de aprendizaje. Otras 8 preguntas solicitaban al alumnado valorar su percepción sobre la contribución a su proceso de aprendizaje de algunos métodos y tecnologías actuales de enseñanza-aprendizaje. En particular, se les requería valorar el uso de los dispositivos electrónicos (Tablet, Smartphone y PC) y de las redes sociales (WhatsApp, Facebook y Twitter, entre otras). El resto de las preguntas se referían a sus características generales del alumnado (sexo, edad, Grado en el que están matriculados e interés por los estudios que están cursando, entre otras). En la mayor parte de las preguntas los alumnos tenían que valorar según una escala de 1 a 5 puntos.

Para calcular el tamaño de la muestra se utilizó la siguiente expresión:

\footnotetext{
2 Este trabajo se enmarca un Proyecto de Innovación Docente denominado "Trabajo colaborativo basado en roles de responsabilidad y herramientas digitales opcionales con retroalimentación entre distintos cursos de GRADO" financiado por la Universidad del País Vasco (UPV/EHU).
} 


$$
n=\frac{N P q}{\frac{(N-1) B^{2}}{Z^{2}}+P q}
$$

Donde:

$\mathrm{n}$ : tamaño de la muestra; $\mathrm{N}$ : tamaño de la población; $\mathrm{P}$ : proporción muestral (utilizamos $\mathrm{p}=\mathrm{q}=0.5$ para garantizar el mayor tamaño de muestra posible); $\mathrm{q}: 1-\mathrm{P}$; B: error permisible (5\%); Z: Valor de z para un nivel de significado dado (1.96, para un nivel de significación del 95\% y 2.47 al $99 \%)$.

El tamaño de la muestra (n) resultante es 264 para un nivel de significación del $95 \%$ y de 354 para un nivel de significación del 99\%.

Se recogieron un total de 358 respuestas. El $47.8 \%$ de los estudiantes que respondieron eran varones y el $52.2 \%$ mujeres. Casi la mitad de los/las participantes (46.9) tenían 18 años en el momento de responder al cuestionario. El 19\% tenían 19 años, el 11.7\% tenían 20 años, el $10.3 \%$, 21 años y el 12\% tenían más de 22 años. El 48\% de los/las encuestadas estaban cursando el Grado en Administración y Dirección de Empresas y el 22.3\% el Grado de Economía. El resto se encontraban matriculados en los Grados de Fiscalidad y Administración pública (6.7\%), Marketing (7\%), Finanzas y Seguros (8\%) y Doble grado en Derecho y Administración (8\%). La tabla 1 presenta un resumen de las características de la muestra.

\begin{tabular}{|c|c|}
\hline Número de respuestas & 358 \\
\hline \multicolumn{2}{|l|}{ Grado } \\
\hline - Administración y Dirección de Empresas & $48.0 \%$ \\
\hline - Economía & $22.3 \%$ \\
\hline — Fiscalidad y Administración Pública & $6.7 \%$ \\
\hline - Marketing & $7.0 \%$ \\
\hline - Finanzas y Seguros & $8.0 \%$ \\
\hline - Doble grado en Derecho y Administración & $8.1 \%$ \\
\hline \multicolumn{2}{|l|}{ Sexo } \\
\hline - Mujeres & $52.2 \%$ \\
\hline - Varones & $48.8 \%$ \\
\hline
\end{tabular}

Tabla 1. Características de la muestra

\subsection{Validez y fiabilidad del cuestionario}

Para la elaboración del cuestionario se creó un focus group, formado por diez personas (4 alumnos y 6 profesores) y moderado por un profesor, en el que se debatieron aspectos relevantes para la formulación de las preguntas del cuestionario. Con base en una revisión de la literatura $y$, teniendo presente el objetivo de este trabajo, la experiencia del profesorado implicado en este Proyecto y las aportaciones del focus group se elaboró un cuestionario.

La comprobación de la validez del cuestionario se ejecutó en varias fases. En la primera, se realizó una valoración del cuestionario por parte de un equipo de profesores con amplia 
experiencia docente en la Universidad, quienes verificaron que la selección y redacción de los ítems era clara y conducente a una respuesta lo más precisa y relevante posible. En la segunda fase, se trasladó el cuestionario a un grupo piloto de estudiantes de la Facultad de Economía y Empresa, con quienes se debatieron aspectos relativos al ordenamiento interno del cuestionario, la longitud o la compresión de las cuestiones y se plantearon algunas modificaciones. Finalmente, se definió el modelo final teniendo en cuenta las aportaciones realizadas en las fases anteriores.

El siguiente paso consistió en evaluar la fiabilidad del cuestionario. La fiabilidad se refiere a la confianza que se concede a los datos que se obtienen con el mismo y está relacionada con la coherencia o consistencia interna y la precisión de las medidas recopiladas (Lacave et al., 2015).En nuestro caso la evaluación de la consistencia interna del cuestionario se realizó mediante el cálculo de diferentes estadísticos: alfa de Cronbach $(\alpha)$, lamda-2 de Guttman $(\lambda-2)$ e índice de Mokken. Los dos primeros se computaron en SPSS 24.0 y el último se calculó utilizando STATA 14.0.

\begin{tabular}{|l|l|}
\hline Estadístico & 0.683 \\
\hline Alfa de Cronbach: & 0.685 \\
\hline Lamda-2 de Guttman: . & 0.360 \\
\hline Índice de Mokker
\end{tabular}

Tabla 2. Estadísticos de consistencia interna

El coeficiente $\alpha$ de Cronbach, ampliamente utilizado como prueba de fiabilidad, es una media de las correlaciones entre las variables que forman parte de una escala. Sus valores oscilan entre 0 y 1 y se considera que el valor de fiabilidad en una investigación exploratoria debe ser igual o mayor a 0.6 (Huh, Delorme y Reid, 2006). El coeficiente $\alpha$, no obstante, ha sido cuestionario debido a que tiene supuestos muy estrictos: unidimensionalidad, errores no correlacionados y tau-equivalencia esencial de todos los elementos ${ }^{3}$ (Sijtsma y van der Ark, 2015; Trizano-Hermosilla y Alvarado, 2016). Estos supuestos, en la mayoría de los casos, no se cumplen, por lo que el $\alpha$ tiende a sobre o subestimar la verdadera fiabilidad.

Una alternativa al $\alpha$ de Cronbach es el $\lambda-2$ de Guttman. Este último indica qué parte de la varianza se debe a valores verdaderos. Si los estudiantes encuestados difieren significativamente en sus características, $\lambda-2$ será alto y el error será bajo. Finalmente, el método de Mokken es una generalización no paramétrica de la escala de Guttman que se aplica para determinar la dimensionalidad de pruebas o escalas, y permite considerar la confiabilidad, sin depender del alfa de Cronbach. Se considera adecuado a partir de 0.30.

Los resultados que obtenemos en los tres estadísticos sugieren que la fiabilidad del cuestionario es adecuada.

\section{METODOLOGÍA}

La metodología utilizada consiste en la aplicación de técnicas estadísticas multivariantes y análisis clúster. La técnica multivalente empleada es el Análisis de Correspondencias Múltiples (ACM). El ACM es un tipo de Análisis Factorial que está orientado a la descripción y análisis de bases de datos en las que los individuos están definidos por variables cualitativas. Está particularmente bien adaptada para estudiar encuestas con respuestas múltiples, como es el

\footnotetext{
${ }^{3}$ La equivalencia de tau esencial significa que todas las covarianzas entre los elementos deben ser idénticas
} 
caso que nos ocupa. El ACM puede ir acompañado de un análisis complementario, el análisis Clúster, que permite obtener clases o grupos de individuos con características homogéneas.

El ACM requiere la selección de un conjunto de variables denominadas activas, que sirven para discriminar entre el universo de individuos. La selección de variables activas se ha realizado teniendo presente el objetivo de nuestro trabajo y la información disponible. Las variables activas consideradas se refieren: dispositivo electrónico más comúnmente utilizado (PC, Tablet o móvil), herramientas de internet más usadas (Facebook, Twitter, Whatsapp), y a la contribución a su aprendizaje de diferentes métodos de aprendizaje (tareas individuales, tareas en grupo, clases teóricas, clases prácticas, tareas en el aula y tareas fuera del aula). Los alumnos valoraban en una escala Likert de 5 puntos todas las cuestiones, a excepción de las dos primeras. La escala Likert original de 5 puntos de se ha recodificado en 3 puntos con objeto de discriminar más eficientemente los individuos. La Tabla 3 recoge las preguntas que han servido de base para las variables activas.

\begin{tabular}{|c|c|c|}
\hline Soporte & $\begin{array}{l}\text { ¿Cuál es el soporte informático más } \\
\text { utilizado para descargar datos, consultar } \\
\text { información, crear fórmulas y/o hacer } \\
\text { gráficos relacionados con el curso? }\end{array}$ & 1. PC; 2. Tablet; 3. Móvil \\
\hline Herram & $\begin{array}{l}\text { ¿Cuál es la herramienta que } \\
\text { preferirías usar en clase para intercambiar } \\
\text { información con tus compañeros? }\end{array}$ & $\begin{array}{l}\text { 1. Facebook; 2. Twitter; } \\
\text { 3. WhatsApp }\end{array}$ \\
\hline Individ & $\begin{array}{l}\text { Valora la contribución del trabajo individual en } \\
\text { tu proceso de aprendizaje }\end{array}$ & $\begin{array}{l}1 \text { Nada/Poco; 2. Bastante; } 3 . \\
\text { Mucho }\end{array}$ \\
\hline Grupo & $\begin{array}{l}\text { Valora la contribución del trabajo en grupo en } \\
\text { tu proceso de aprendizaje }\end{array}$ & $\begin{array}{l}1 \text { Nada/Poco; 2. Bastante; } 3 . \\
\text { Mucho }\end{array}$ \\
\hline Teórica & $\begin{array}{l}\text { Valora la contribución de las clases teóricas o } \\
\text { magistrales en tu proceso de aprendizaje }\end{array}$ & $\begin{array}{l}1 \text { Nada/Poco; 2. Bastante; } 3 . \\
\text { Mucho }\end{array}$ \\
\hline Práctica & $\begin{array}{l}\text { Valora la contribución de las clases prácticas } \\
\text { desarrolladas por el profesor en tu proceso de } \\
\text { aprendizaje }\end{array}$ & $\begin{array}{l}1 \text { Nada/Poco; 2. Bastante; } 3 . \\
\text { Mucho }\end{array}$ \\
\hline Aula & $\begin{array}{l}\text { Valora la contribución de la resolución de } \\
\text { ejercicios en el aula en tu proceso de } \\
\text { aprendizaje }\end{array}$ & $\begin{array}{l}1 \text { Nada/Poco; 2. Bastante; } 3 . \\
\text { Mucho }\end{array}$ \\
\hline Fuera & $\begin{array}{l}\text { Valora la contribución de la resolución de } \\
\text { ejercicios fuera del aula en tu proceso de } \\
\text { aprendizaje }\end{array}$ & $\begin{array}{l}1 \text { Nada/Poco; 2. Bastante; } 3 . \\
\text { Mucho }\end{array}$ \\
\hline Impacto & $\begin{array}{l}\text { ¿Qué impacto crees que puede tener sobre el } \\
\text { resultado académico la incorporación de } \\
\text { dispositivos móviles y redes sociales? }\end{array}$ & $\begin{array}{l}1 \text { Negativo; } 2 . \text { No afecta; } 3 . \\
\text { Positivo }\end{array}$ \\
\hline
\end{tabular}

Tabla 3. Preguntas y variables activas

Además de las variables activas, también se pueden incorporar variables denominadas ilustrativas, que sirven para completar la descripción proporcionada por las variables activas. Hemos seleccionado el sexo, la edad y la calificación esperada como variables ilustrativas.

Para comprobar la conveniencia de aplicar el ACM, se ha evaluado la significatividad del modelo factorial. Para ello, hemos calculado la prueba de esfericidad de Bartlett y el índice de 
Kaiser-Meyer-Olkin (Tabla 5). La prueba de Bartlett evalúa la correlación entre las variables objeto de estudio. El valor obtenido (190; Sig. 0,000) muestra que podemos rechazar la hipótesis de no correlación entre las variables y afirmar que hay al menos un factor común que motiva la aplicación del análisis factorial. El índice de Kaiser-Meyer-Olkin (KMO) es una medida de adecuación muestral de las variables. El valor de esta medida $(0,707)$ excede sensiblemente el valor mínimo sugerido para aplicar el análisis factorial.

Medida de adecuación muestral de Kaiser-Meyer-

0.707 Olkin

Prueba de esfericidad de Bartlett

\begin{tabular}{lr|r}
$\begin{array}{l}\text { Approx. } \\
(21)\end{array}$ & Chi-Square & 190 \\
\hline Sig. & & 0,0000 \\
\hline
\end{tabular}

El índice de KMO varía entre 0 y 1 . Se consideran adecuados los valores superiores a 0,5.

Tabla 4. KMO y Prueba de Barlett

\section{RESULTADOS}

\subsection{Resultados estadísticos}

La tabla 4 presenta una descriptiva de las variables utilizadas en el análisis empírico. Los resultados sugieren que el uso de dispositivos móviles y redes sociales (a excepción del WhatsApp) no está muy extendido entre los estudiantes encuestados en sus actividades formativas. Es de notar la preferencia del alumnado por las actividades desarrolladas dentro del aula y de tipo tradicional, como las clases teóricas y los ejercicios o tareas dentro del aula. Pese a que el uso de dispositivos móviles y las redes sociales (a excepción del WhatsApp) no está muy extendido entre los estudiantes para tratar cuestiones académicas, más de la mitad (59\%) considera que la incorporación de estas herramientas podría tener un impacto positivo en sus resultados.

\begin{tabular}{|c|c|c|c|}
\hline \multirow[t]{2}{*}{ Soporte } & PC & Tablet & Smartphone \\
\hline & 82.9 & 12.0 & 5.1 \\
\hline \multirow[t]{2}{*}{ Herramientas } & Facebook & Twitter & WhatsApp \\
\hline & 2.2 & 1 & 97 \\
\hline Contribución al proceso enseñanza-aprendizaje & $\begin{array}{c}1 . \\
\text { Nada/Poco }\end{array}$ & $\begin{array}{c}2 . \\
\text { Bastante }\end{array}$ & $\begin{array}{c}3 . \\
\text { Mucho }\end{array}$ \\
\hline Trabajo individual & 0.6 & 33.2 & 66.3 \\
\hline Trabajo en grupo & 11.2 & 36.9 & 51.9 \\
\hline Clase teórica & 8.4 & 32.9 & 58.7 \\
\hline Clase práctica & 3.3 & 20.7 & 76.0 \\
\hline Ejercicios dentro del aula & 3.1 & 21.5 & 75.4 \\
\hline Ejercicios fuera del aula & 44.5 & 41.3 & 47.2 \\
\hline \multirow[t]{2}{*}{$\begin{array}{l}\text { Impacto sobre el resultado académico de la } \\
\text { incorporación de dispositivos móviles y redes } \\
\text { sociales }\end{array}$} & Negativo & $\begin{array}{l}\text { No } \\
\text { afecta }\end{array}$ & Positivo \\
\hline & 4.5 & 36.0 & 59.5 \\
\hline
\end{tabular}

Tabla 5. Descripción de las variables activas (en \%) 


\subsection{Resultados del ACM}

En esta sección se presentan e interpretan los resultados más representativos del ACM. La Tabla 4 presenta los tres primeros valores propios. El primer factor explica el $14.2 \%$ de la varianza, el segundo eje el $10.1 \%$ y el tercero, el $9.2 \%$. Los dos primeros ejes (factores) explican el $24.7 \%$ de la varianza total, lo que puede considerarse como un resultado muy satisfactorio en este tipo de análisis. Los tres ejes explican conjuntamente el $33.8 \%$ de la varianza total.

\begin{tabular}{|c|c|c|c|}
\hline & Valor Propio & Porcentaje & P. Acumulado \\
\hline $\mathbf{1}$ & 0.2373 & 14.16 & 14.16 \\
\hline $\mathbf{2}$ & 0.1650 & 10.12 & 24.68 \\
\hline $\mathbf{3}$ & 0.1496 & 9.18 & 33.85 \\
\hline
\end{tabular}

Tabla 6. Valores propios de los primeros factores

Las variables que contribuyen a la formación de los dos primeros ejes (factores) aparecen representadas en el Gráfico 1. Las variables relacionadas con el interés por la asignatura (Interés), y con los métodos de enseñanza son las que más contribuyen a la inercia del primer factor. En particular, destacan los métodos de enseñanza más tradicionales (clases teóricas, las clases prácticas, tareas en el aula y tareas desarrolladas fuera del aula). Las modalidades de todas estas variables se ordenan en el primer factor de mayor a menor, constituyendo lo que se denomina un efecto talla. Este primer eje, por tanto, permite una primera clasificación del alumnado en cuanto a su opinión sobre la contribución a su aprendizaje (e interés por la asignatura) de cada uno de los métodos de enseñanza.

El segundo eje pone de relieve una segunda clasificación del alumnado. Las variables que más contribuyen a su formación son las relativas al dispositivo electrónico ( $P C$, Tablet o Smartphone) y a las redes sociales más utilizadas. El conjunto de estudiantes puede ordenarse en relación con su preferencia y uso de estos dispositivos y herramientas oponiendo los/as estudiantes que usan la red social Facebook a los que usan Twitter y los que usan Tablet a los prefieren el uso de su Smartphone. La preferencia por el PC y el uso de WhatsApp no presenta diferencias entre el alumnado, por ello están situados en el origen del plano factorial.

Por otro lado, se puede observar que los estudiantes con una mejor percepción de la contribución que hacen los distintos métodos de enseñanza a su adquisición de competencias tienden a usar como soporte electrónico preferente la Tablet. Además, declaran utilizar en mayor medida que el resto de estudiantes, las herramientas de internet Twitter y Facebook. El uso de Smartphone aparece más conectado con el alumnado con menor valoración de la contribución que hacen los diferentes métodos de enseñanza aprendizaje a su formación. 


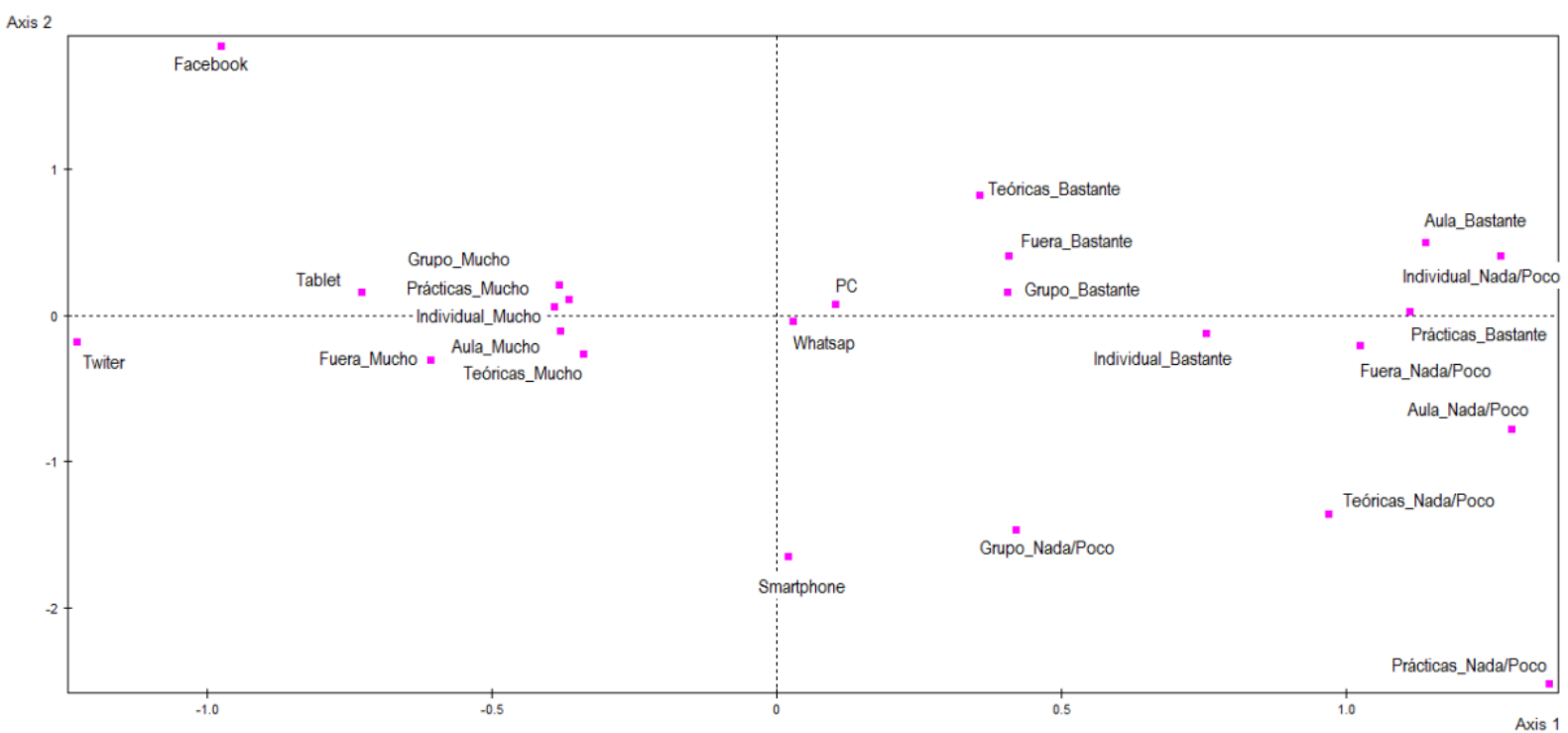

Gráfico 1. Representación de las variables activas en el primer plano factorial

Las variables ilustrativas aparecen representadas en el Gráfico 2 junto con las variables activas. Se observa que algunas diferencias en el comportamiento de hombres y mujeres. Estas últimas muestran relativamente un mayor interés en las asignaturas y otorgan una mayor valoración a los métodos de enseñanza como motor de su aprendizaje. Las expectativas de altas calificaciones aparecen asociadas a los/las estudiantes más interesados en la asignatura, que utilizan en mayor medida la Tablet y que están más familiarizados con el uso de Facebook y Twitter.

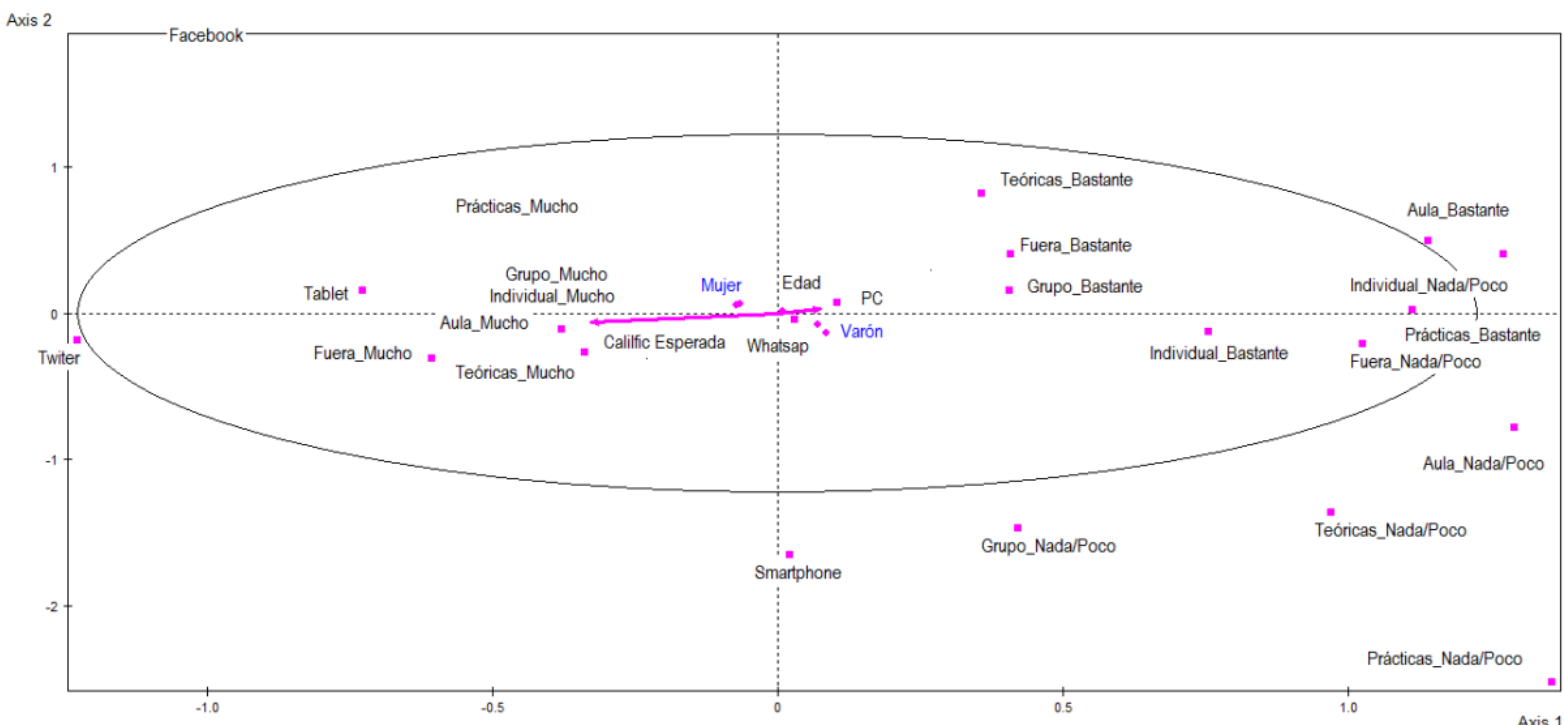

Gráfico 2. Representación de las variables activas e ilustrativas en el primer plano factorial

Con base en esta disposición de las variables en el Gráfico 3 se representan la distribución en el primer plano factorial de todos los individuos que han respondido a la encuesta. Se observa 
que los estudiantes se distribuyen en los cuatros cuadrantes del plano factorial, poniendo de manifiesto la existencia de una gran heterogeneidad entre el colectivo.

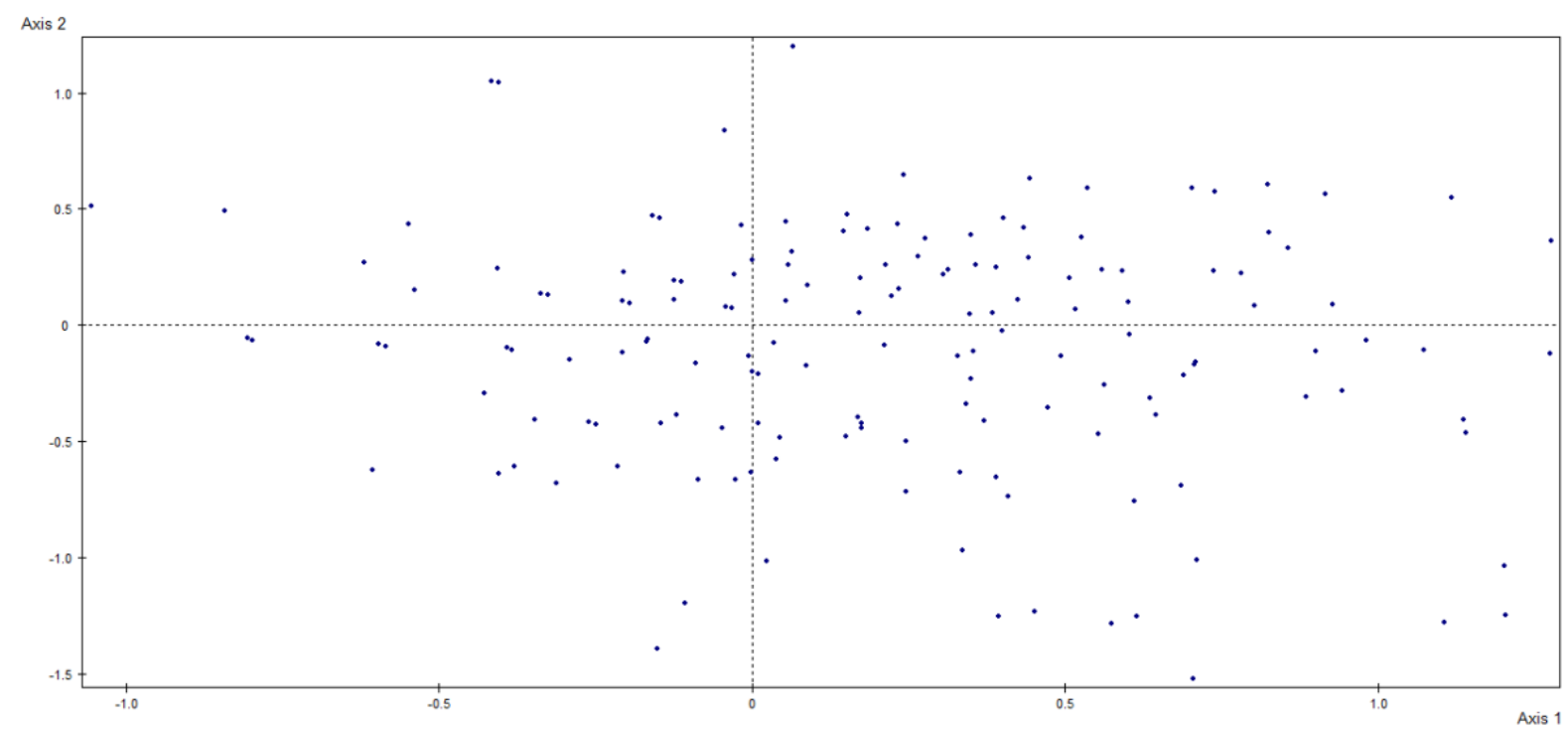

Gráfico 3. Distribución de los individuos en el primer plano factorial

\subsection{Resultados del Análisis Clúster}

El análisis clúster permite agrupar a los estudiantes en grupos o clases diferentes. Este análisis se ha realizado a partir de la información obtenida en el ACM con los tres primeros factores extraídos. Los/as estudiantes que pertenecen a un mismo grupo tienen características homogéneas en cuanto a su percepción de los diferentes métodos de enseñanza y al uso de herramientas informáticas y de internet. Los/as estudiantes que pertenecen a grupos diferentes presentan rasgos distintos. Es preciso apuntar que los/as estudiantes dentro de un mismo grupo no comparten necesariamente todas las variables que caracterizan al grupo, aunque sí una parte estadísticamente significativa de ellas.

En nuestro análisis clúster hemos identificado una tipología sobre usos y beneficios de los dispositivos electrónicos y las redes sociales en los procesos de enseñanza-aprendizaje entre el alumnado de la Facultad, compuesto por tres grupos de estudiantes bien definidos, que hemos denominado de menor a mayor uso de la tecnología móvil y de las redes sociales: Usuarios básicos, Usuarios Moderados y Usuarios Intensivos (ver Dendograma en el Gráfico 4). 


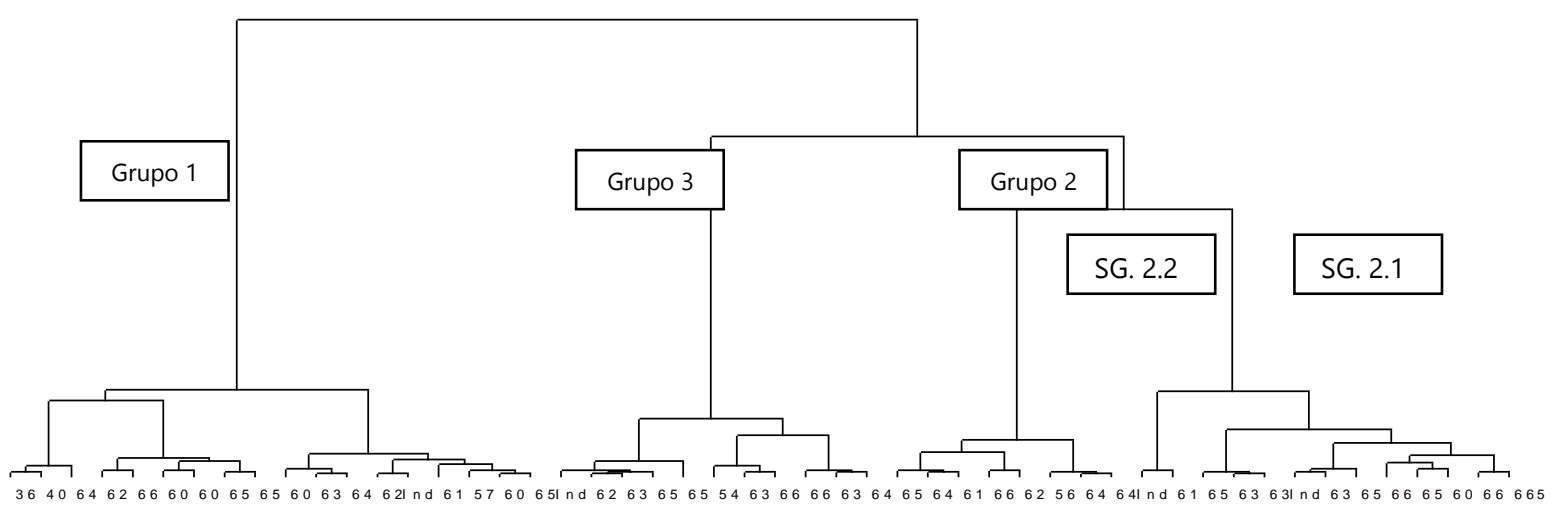

Gráfico 4. Dendograma

El grupo 1 (Usuarios básicos) contiene al 15.08\% del alumnado. El 66.7\% de los/as estudiantes de este grupo considera que las tareas realizadas en grupo contribuyen poco o nada a su formación (frente al 11.2\% que caracteriza al conjunto de los individuos encuestados). La misma opinión tiene el $14.8 \%$ del alumnado de este grupo sobre las clases prácticas (frente al $3.3 \%$ en el conjunto de alumnos/as). Las clases teóricas y el trabajo individual son los métodos que más contribuyen a su formación para el $83.3 \%$ y $50 \%$ de los/las integrantes de este grupo, respectivamente. Estas proporciones son del $58.7 \%$ y $33.2 \%$, en el conjunto de individuos estudiado, respectivamente.

Para $27 \%$ de los/las estudiantes de este grupo el Smartphone es el dispositivo preferido y con el que mejor se manejan (frente al $5.0 \%$ en el conjunto del alumnado estudiado). En este grupo se concentra el $83.3 \%$ de estudiantes que consideran el Smartphone como soporte principal para sus actividades relacionadas con las TICS. Este conjunto de estudiante no destaca por el uso de ninguna red social en particular Además, el 50\% de los estudiantes de este grupo considera que la incorporación de estas tecnologías no va a influir en su resultado académico.

En definitiva, este grupo parece mostrar preferencia por las formas más tradicionales de enseñanza-aprendizaje (clases teóricas y trabajo individual), muestra un interés muy bajo en el uso de redes sociales (más allá del WhatsApp), su soporte informático preferido es el Smartphone y considera que sus resultados no se verían afectados con el uso de estas herramientas en las aulas.

El grupo 2 (Usuarios Moderados) agrupa el 29.3\% del alumnado. El 58.1\% de los/as estudiantes de este grupo declara que los ejercicios en el aula contribuyen bastante a su formación mientras que para el $31.4 \%$ los realizados fuera del aula no contribuyen a su aprendizaje. Para el conjunto de los estudiantes estos porcentajes son del $21.5 \%$ y $11.4 \%$, respectivamente. El alumnado de este grupo considera bastante relevante la contribución que las prácticas hacen a su capacitación. Así opina el $44.8 \%$ del alumnado de este grupo frente al $20.7 \%$ como media en el conjunto de encuestados. Para el $94.3 \%$ de los/as estudiantes de este grupo el dispositivo más usado es el PC (frente al $82.9 \%$ en el conjunto del alumnado estudiado). Cabe señalar que en este grupo se concentra el $33.3 \%$ de estudiantes que manifiestan su preferencia por el PC como soporte informático para desarrollar sus actividades. Este grupo tampoco destaca por el uso de ninguna red social en particular.

Dentro de este grupo, como se puede apreciar en el dendograma (Gráfico 4), es posible distinguir dos subgrupos. Por un lado, un conjunto de estudiantes que consideran que la mayoría de los métodos de enseñanza-aprendizaje utilizados (ejercicios dentro y fuera del aula, 
trabajos individuales y clases teóricas y prácticas) contribuyen bastante a su aprendizaje. Entre el $48 \%$ y $69 \%$ de los/as alumnos/as de este grupo tienen esa opinión favorable sobre dichos métodos de enseñanza aprendizaje. En el conjunto de la muestra estos porcentajes oscilan entre el $20 \%$ y $41 \%$. El $84.8 \%$ de los estudiantes de este subgrupo muestran su preferencia por el PC cuando se trata de descargar datos, crear fórmulas y hacer gráficos. Por otro lado, se identifica otro subgrupo en el que sus participantes consideran que la mayoría de los métodos de enseñanza aprendizaje utilizados contribuyen poco o nada a su formación. Este subgrupo utiliza como soportes informáticos el PC, Tablet y Smartphone.

El grupo 3 (Usuarios Intensivos) contiene al 55.59\% de los/as estudiantes. El $95.5 \%$ de los estudiantes de este grupo consideran que los ejercicios realizados en el aula hacen una contribución muy importante a su formación académica (frente al $75.4 \%$ que tienen esa opinión en el conjunto de individuos analizado). También un alto porcentaje de estudiantes valora muy positivamente la contribución de otros métodos como las clases prácticas $(94.9 \%$ frente al $75.8 \%$ en el conjunto de estudiantes analizado), los trabajados individuales (85.4\% frente al $66.2 \%$ ) y los ejercicios fuera del aula (65.8\% frente al $47.2 \%)$.

Para el $17.1 \%$ la Tablet es el soporte informático más utilizado (frente al $12 \%$ en el conjunto de la muestra). El uso de WhatsApp para intercambiar información con los compañeros/as, sin embargo, es utilizado por debajo de la media. Una proporción significativa de estudiantes de este grupo considera que sus resultados mejorarían con la incorporación de estas herramientas.

En este grupo se concentra el $79.1 \%$ de los estudiantes analizados que expresan una clara preferencia por el uso de la Tablet para descargar datos, crear fórmulas y hacer gráficos. También se encuentran en este grupo casi el 70\% de los/as alumnos/as que consideran muy importante para su formación la mayoría de los métodos de enseñanza- aprendizaje utilizados (actividades desarrolladas dentro del aula, las clases prácticas, entre otras).

\section{DISCUSIÓN Y CONCLUSIONES}

Este trabajo examina el grado de penetración de los dispositivos móviles (smartphones y tables) y de las redes sociales entre los estudiantes de la Facultad de Economía y Empresa de la Universidad del País Vasco (UPV/EHU), y analiza la percepción que tiene el alumnado respecto a la contribución que tienen dichas herramientas en los procesos y resultados de su aprendizaje.

En primer lugar, hay que destacar que, el uso de las Tics es valorada positivamente por los alumnos de la Facultad en su desarrollo académico, por las potencialidades que presentan en su rendimiento académico. Esta percepción va en la línea de la obtenida en los trabajos de Morales et al., 2015.

Los resultados, no obstante, también sugieren que, en el conjunto de estudiantes analizado, el uso de dispositivos móviles y redes sociales no está muy extendido a la hora de interactuar con el contenido del curso y colaborar con otros estudiantes o profesores. Para una proporción significativa del alumnado estas herramientas quedan fuera del ámbito de la educación universitaria formal, quedando restringidas al uso privado (relaciones sociales y ocio). Los estudios realizados por Cabezas et al. (2014), Sánchez- Rodríguez et al. (2015), y Prendes et al. (2017), alcanzan similares conclusiones en relación al uso que los estudiantes universitarios 
hacen de las TICs. Estos resultados difieren de los encontrados en otros trabajos (Heath et al., 2005; Gikas y Grant, 2013; Vega-Hernández et al., 2018) en los que se mostraba una amplia interacción del alumnado con los materiales del curso y con el resto de estudiantes. Asimismo, se aprecian diferencias en cuanto al tipo de dispositivo y red social preferida por el alumnado. Así, el acceso a contenidos e información relacionada con el curso parece realizarse a través de la Tablet, mientras que el uso del móvil está más asociado a las relaciones personales, siendo el WhatsApp la herramienta más generalizada.

En segundo lugar, el estudio permite elaborar una tipología del alumnado de la Facultad con relación a los usos y los beneficios de las redes sociales en el proceso de enseñanza-aprendizaje. Así, se han identificado tres grupos claramente diferenciados. Por un lado, el grupo de usuarios intensivos de estas herramientas, que constituye más de la mitad del alumnado. Por otro, el grupo de usuarios básicos (15\% del alumnado), que parece mostrar preferencia por las formas más tradicionales de enseñanza-aprendizaje (clases teóricas y trabajo individual) y muestra un interés muy bajo en el uso de redes sociales. El resto de alumnos se manifiesta como usuarios moderados.

En definitiva, en el ámbito educativo todavía hay un número significativo de alumnos que presentan cierta resistencia a emplear las herramientas web 2.0 en su proceso de aprendizaje. Este aspecto es susceptible de una cierta reflexión, en tanto que parece evidente que nuestra realidad presente y futura es la de un mundo globalizado e interconectado, donde es importante que los estudiantes desarrollen habilidades digitales como parte de su formación. Favoreciendo así el desarrollo de competencias como el de trabajo autónomo, la capacidad crítica y reflexión al seleccionar, tratar y utilizar la ingente información que tienen a su disposición

No obstante, también es cierto que el uso de estas herramientas en la dinámica formativa se enfrenta todavía a diversos obstáculos. Por un lado, si bien la práctica totalidad de los estudiantes dispone de dispositivos móviles en las aulas, se necesita un consenso entre estudiantes y entre estudiantes y docentes sobre las redes sociales más idóneas, así como una infraestructura adecuada en el centro (Wifi libre, conexiones eléctricas adecuadas, etc.). Por otro lado, la incorporación de la tecnología en la formación universitaria también pasa por un adecuado adiestramiento del profesorado. Es preciso, por tanto, avanzar en el conocimiento sobre las competencias y las actitudes del profesorado hacia la incorporación y uso de dispositivos móviles y redes sociales en su actividad docente. Finalmente, si aceptamos que el uso de dispositivos móviles y redes sociales tiene un efecto positivo en el proceso-aprendizaje de los estudiantes universitarios, tal y como han destacado algunos autores (Dunlap y Lowenthal, 2009; Ophus y Abbitt, 2009; Selwyn, 2010; Gao et al., 2012, Sadaf et al., 2012) nuestros resultados sugieren que es necesario diseñar e implementar una estrategia específica orientada tanto al alumnado como al profesorado. Los trabajos de Lareki et al. (2010) y Sánchez-Rodríguez et al. (2015), entre otros, ponen de hecho el énfasis en la falta de competencia digital tanto del profesorado como de los alumnos en los procesos de enseñanzaaprendizaje

Por último, este trabajo sugiere algunas líneas de investigación futuras. Por un lado, orientadas a valorar los cambios en las percepciones del alumnado una vez que se hayan implementado determinadas estrategias para extender el uso de las redes sociales en el proceso de formación. Por otro lado, resulta conveniente conocer el uso y la opinión del colectivo de profesores sobre la aplicación de estas nuevas tecnologías en el proceso de enseñanza-aprendizaje. Detectar posibles carencias de formación en el manejo de las mismas en ambos colectivos parece algo 
obligado con el fin de obtener el máximo rendimiento de las herramientas digitales al servicio de la educación universitaria. Asimismo, habría que analizar qué red social es más conveniente emplear para conseguir los objetivos perseguidos en la educación universitaria.

\section{REFERENCIAS}

Asociación de Investigación de Medios de Comunicación (AIMC) (2017): Navegantes en la Red. Recuperado de http://www.aimc.es/otros-estudios-trabajos/navegantes-la-red/

Bernal, C. y Angulo, F. (2012). Interacciones de los jóvenes andaluces en las redes sociales. Comunicar, 20 (40), 25-30. Recuperado de: http://dx.doi.org/10.3916/C40-2013-02-02

Cabezas, M., Casillas, S. y Pinto, A.M. (2014): "Percepción de los alumnos de Educación Primaria de la Universidad de Salamanca sobre su competencia digital". Edutec. Revista Electrónica de Tecnología Educativa, 48, junio. Recuperado de: https://doi.org/10.24320/redie.2017.19.4.1369

Cataldi, Z., Méndez, P., Dominighini, C. y Lage, F.J (2012): Dispositivos móviles en educación superior y entornos personalizados de aprendizaje. WICC, XIV Workshop de Investigaciones en Ciencias de la Computación. Recuperado de: http://sedici.unlp.edu.ar/handle/10915/19437

Contreras Cázarez, C. R. y Campa Álvarez, R. (2017). Caracterización del perfil de los estudiantes de secundarias en el acceso y uso de internet a partir de las TIC http://www.edutec.es/revista/index.php/edutec-e/article/view/941. Edutec, Revista Electrónica de Tecnología Educativa, 61. Recuperado de: http://www.edutec.es/revista/index.php/edutec-e/article/view/941

Dunlap, J. C. y Lowenthal, P. R. (2009). Tweeting the night away: Using Twitter to enhance social Education", Journal of Information Systems Education, 20 (2), 129-135. Recuperado de: https://doi.org/110.1177/0273475315586061

Ebner, M., Lienhardt, C., Rohs, M. y Meyer, I. (2010). Microblogs in higher education- a chance to facilitate informal and process-oriented learning?, Computers \& Education, 55, 92 100. Recuperado de: https://doi.org/10.1016/j.compedu.2009.12.006

Ebner, M. y Maurer, H. (2009). Can microblogs and weblogs change traditional scientific writing? Future Internet, 1, 47-58. Recuperado de: https://doi.org/10.3390/fi1010047

El-hussein, M. O. M. y Cronje, J. C. (2010). Defining Mobile Learning in the Higher Education Landscape. Research method, 13, 12-21. Recuperado de: http://www.jstor.org/stable/jeductechsoci.13.3.12?seq

Friesen, N. y Lowe, S. (2011). The questionable promise of social media foreducation: Connective learning and the commercial imperative. Journal of Computer Assisted Learning, 28(3), 183-194. Recuperado de: https://doi.org/10.1111/j.13652729.2011.00426.x 
Gao, F., Luo, T. y Zhang, K. (2012). Tweeting for learning: a critical analysis of research on microbloging in education published in 2008-2011, British Journal of Education Technology, 43(5), 783-801. Recuperado de: https://doi.org/10.1111/j.14678535.2012.01357.x

Gikas, J. y Grant, M.M. (2013). Mobile computing devices in higher education: student perspectives on learning with cellphones, smartphones and social media. Internet and Higher education, 19, 18-26. Recuperado de: https://doi.org/110.1016/j.iheduc.2013.06.002

Gisbert Cervera, M., Espuny Vidal, C. y González Martínez, J. (2012). Cómo trabajar la competencia digital con estudiantes universitarios. Recuperado de http://www.edutic.ua.es/wp-content/uploads/2012/06/La-practicaeducativa 157 174-CAP14.pdf

Grosch, M., Berger, R., Gidion, G. y Romeo, M. (2014). Which media services do students use in fact? Results of an international empirical survey, Procedia - Social and Behavioral Science, 141,795-806. Recuperado de: https://doi.org/10.1016/j.sbspro.2014.05.139

Grosseck, G. y Holotescu, C. (2008). Can we use twitter for educational activities?, Paper presented at The 4th International Scientific Conference. Recuperado de: http://dx.doi.org/10.1177/1469787411429190

Heath, B.P., Herman, R.L., Lugo, G.G., Reeves, J.H., Vetter, R.J. y Ward, C.R. (2005). Project Numina: Enhancing student learning with handheld computers. IEEE Computer Society. Recuperado de: http://dx.doi.org/10.1109/MC.2005.199

Hernández Fernández, C. y Alcoceba Hernando, J.A. (2015). Socialización virtual, multiculturalidad y riesgos de los adolescentes latinoamericanos en España, Icono 14(13),116-141. Recuperado de: http://dx.doi.org/10.7195/ri14.v13i2.787

Heath, B. P., Herman, R. L., Lugo, G. G., Reeves, J. H., Vetter, R. J., y Ward, C. R. (2005). Project Numina: Enhancing student learning with handheld computers. IEEE Computer Society, 38(6), 46-52. Recuperado de https://www.computer.org/csdl/mags/co/2005/12/rz086.pdf

Huh J, Delorme D.E y Reid L.N. (2006). Perceived third-person effects and consumer attitudes on preventing and banning DTC advertising, Journal of Consumer Affairs, 40(1), 90-116. Recuperado de: https://doi.org/10.1111/j.1745-6606.2006.00047.x

Humanante-Ramos, P., García-Peñalvo, F. y Conde-González, M. (2017). Entornos personales de aprendizaje móvil: una revisión sistemática de la literatura Mobile Personal Learning Environments: A systematic literature review RIED. Revista Iberoamericana de Educación a Distancia, 20(2), 73-92. Recuperado de: http://dx.doi.org/10.5944/ried.20.2.17692.

IAB (2017). VIII Estudio Anual de Redes Sociales 2017. Interactive Advertising Bureau Spain. Recuperado de https://iabspain.es/?s=redes+sociales 
INJUVE (2012). Jóvenes y nuevas tecnologías, Observatorio del Instituto de la Juventud, marzo. Recuperado de http://www.injuve.es/observatorio/infotecnologia/jovenes-y-nuevastecnologias

Johnson, L. F., Smith, R. S., y Levine, Alan (2005). The Horizon Report. Austin, TX: The New Media Consortium. Recuperado de: http://www.nmc.org/pdf/2005-Horizon-Report.pdf.

Johnson, L. F., Smith, R. S., y Levine, Alan (2009). The Horizon Report. Austin, TX: The New Media Consortium. Recuperado de: http://www.nmc.org/pdf/2009-Horizon-Report.pdf.

Johnson, L. F., Smith, R. S., y Levine, Alan (2014). The Horizon Report. Austin, TX: The New Media Consortium. Recuperado de: http://www.nmc.org/pdf/2014-Horizon-Report.pdf.

Johnson, L. F., Smith, R. S., y Levine, Alan (2015). The Horizon Report. Austin, TX: The New Media Consortium. Recuperado de: http://www.nmc.org/pdf/2015-Horizon-Report.pdf.

Jones, N., Blackey, H., Fitzgibbon, K., y Chew, E. (2010). Get out of MySpace! Computers \& Education, 54(3), 776-782. Recuperado de: https://doi.org/10.1016/j.compedu.2009.07.008

Kaplan, A. M. y Haenlein, M. (2010). Users of the world, unite! The challenges and opportunities of social media. Business Horizons, 53(1), 59-68. Recuperado de: https://doi.org/10.1016/j.bushor.2009.09.003

Lareki, A., Martínez de Morentin, J.I. y Amenabar, N. (2010). Towards an efficient training of university faculty on ICTs, Computer \& Education, 54, 491-497. Recuperado de: http://www.ehu.es/revista-psicodidactica

Morales, M., Trujillo, J.M. y Raso, F. (2015). Percepciones acerca de la integración de las TICs en el proceso de enseñanza-aprendizaje de la universidad, Pixel-Bit. Revista de Medios y Educación, 46, 103-117. doi: http://dx.doi.org/10.12795/pixelbit.2015.i46.07.

Ophus, J.D. y Abbitt, J.T. (2009). Exploring the potential perceptions of social networking systems in University courses, Journal of Online Learning and Teaching, 5(4), 110. Recuperado de: http://dx.doi.org/10.1080/0309877X.2012.726973

Prendes, M.P., Castañeda, L., Gutierrez I. y Román M. (2016). Still far from personal learning: Key aspects and emergent topics about futures professionals' PLES are, Digital Education Review, 29, 15-30, Recuperado de http://greav.ub.edu/der/

Prendes, P., Castañeda, L., Gutierrez, I. y Sánchez, M.M. (2017). Personal learning enviroments in future professionals: nor native or resident, just survivors, International Journal of Information and Education Technology, 7 (3), march. Recuperado de: http://dx.doi.org/10.18178/ijiet.2017.7.3.861

Ramos, A.I., Herrera, J.A. y Ramírez,M.S. (2010). Desarrollo de habilidades cognitivas con aprendizaje móvil: un estudio de casos, Comunicar, Revista Científica de Educomunicación, 34(27), 201-209. Recuperado de: http://dx.doi.org/10.3916/C34$\underline{\text { 2010-03-20 }}$ 
Ramsden, A. (2009). Using Microblogging (Twitter) in your teaching and learning. An introductory guide. Discussion paper. University of Bath. Recuperado de http://opus.bath.ac.uk/15319/1/intro to microblogging 09.pdf

Revuelta, F. (2011). Competencia digital: desarrollo de aprendizajes con mundos virtuales en la Escuela, Edutec. Revista Electrónica de Tecnología Educativa, 37. Recuperado de http://www.edutec.es/revista/index.php/edutec-e/article/view/397/133

Russell, T.L. (2001). The no significant difference phenomenon: a comparative research annotated bibliography on technology for distance education. North Carolina State University Press. Recuperado de: http://www.nosignificantdifference.org/

Sadaf, A., Newby, T. J., y Ertmer, P. A. (2012). Exploring pre-service teachers' beliefs about using web 2.0 technologies in K-12 classroom. Computers \& Education, 59(3), 937-945. Recuperado de https://www.learntechlib.org/p/66461/.

Sanchez-Rodriguez, J., Ruiz-Palmero, J. y Sánchez-Rivas, E. (2015). Uso problemático de las redes sociales en estudiantes universitarios, Revista Complutense de Educación, 26. Num Especial, 159-174. Recuperado de: http://dx.doi.org/10.5209/rev RCED.2015.v26.46360

Schroeder, A., Minocha, S. y Schneidert, C. (2010). The strengths, weaknesses, opportunities and threats of using social software in higher and further education teaching and learning, Journal of Computer Assisted Learning, 26, 159-174. Recuperado de: https:// dx.doi.org/10.1111/j.1365-2729.2010.00347.x

Selwyn, N. (2010). Looking beyond learning: Notes towards the critical study of educational technology. Journal of Computer Assisted Learning, 26(1), 65-73. Recuperado de: http://dx.doi.org/10.1111/j.1365-2729.2009.00338.x

Sijtsma, K. \& Van der Ark, L.A. (2015). Conceptions of reliability revised and practical recommendations. US National Library of Medicine. National Center for Biotechnology Information, U.S. Recuperado de: http://dx.doi.org/10.1097/NNR.0000000000000077

Tess, P.A. (2013). The role of social media in higher education classes (real and virtual) - A literature review. Computers in Human Behavior, 29 (5), A60-A68. Recuperado de http://dx.doi.org/10.1016/j.chb.2012.12.032

Trizano-Hermosilla, I. y Alvarado, J.M. (2016). Best alternatives to Cronbach's alpha reliability in realistic conditions: congeneric and asymmetrical measurement, Frontiers in Psychology, 7, 1-8. Recuperado de: https://dx.doi.org/10.3389/fpsyg.2016.00769

Vega-Hernández, M.C, Patino-Alonso, M.C., y Galindo-Villardón, M.P. (2018). Multivariate characterization of university students using ICT for learning, Computers \& Education, 121, 124-130. Recuperado de: http://dx.doi.org/10.1016/j.compedu.2018.03.004

Para referenciar este artículo:

Altuzarra-Artola, A., Gálvez, C. \& González, A. (2018). Explorando el potencial de los dispositivos 
electrónicos y de las redes sociales en el proceso de enseñanza - aprendizaje de los universitarios. EDUTEC, Revista Electrónica de Tecnología Educativa, 64. Recuperado de: http://dx.doi.org/10.21556/edutec.2018.64.1031 


\section{APÉNDICE}

Cluster $1 / 3$

T.VALUE PROB. ---- PERCENTAGES ---- CHARACTERISTIC IDEN WEIGHT

GRP/CAT CAT/GRP GLOBAL CATEGORIES OF VARIABLES

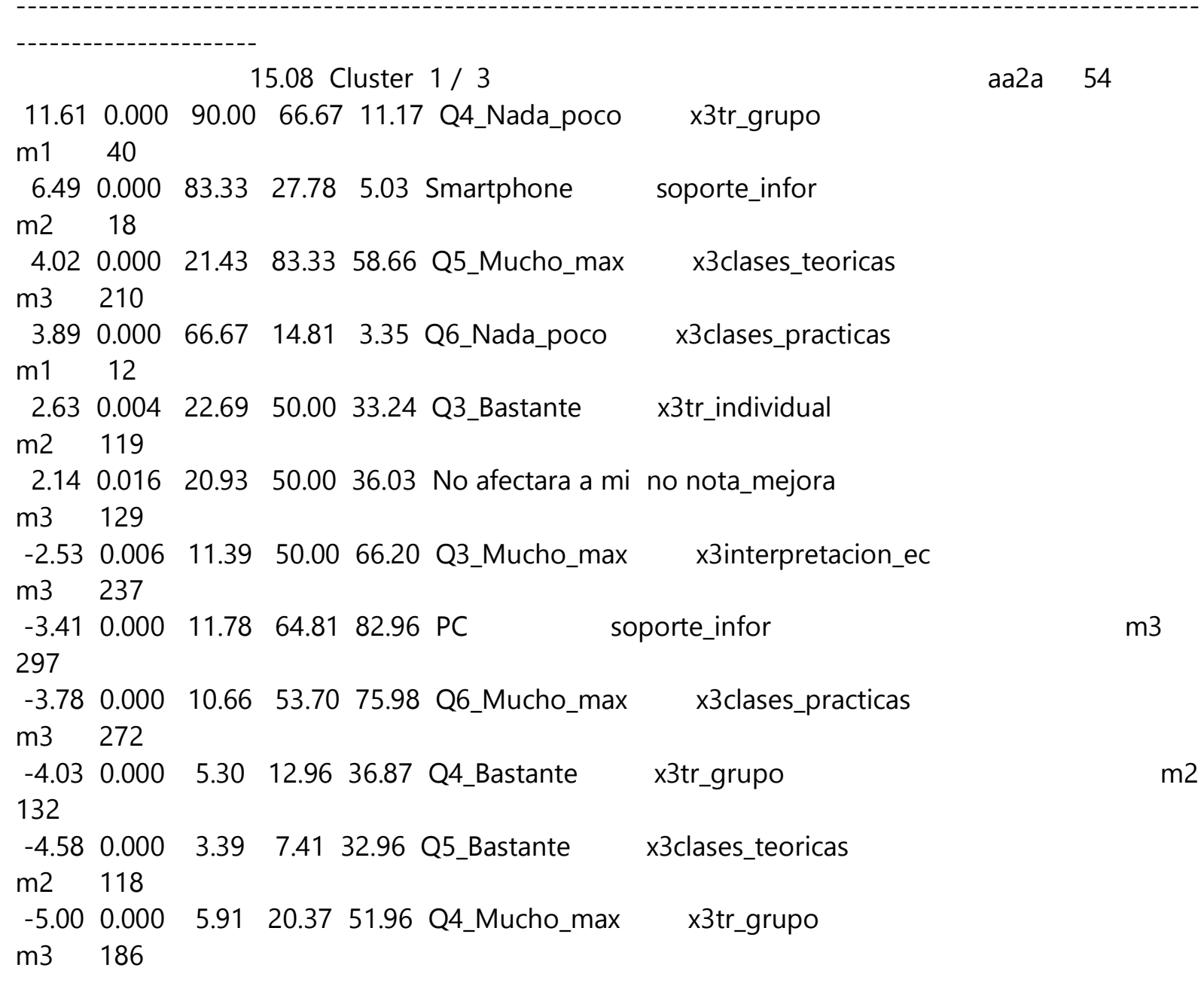

Cluster 2 / 3

T.VALUE PROB. ---- PERCENTAGES ---- CHARACTERISTIC IDEN WEIGHT

GRP/CAT CAT/GRP GLOBAL CATEGORIES OF VARIABLES

29.33 Cluster $2 / 3$

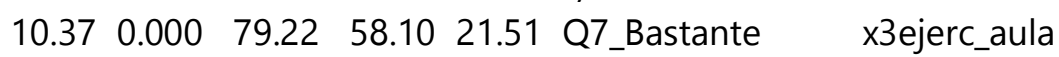
$\mathrm{m} 2 \quad 77$

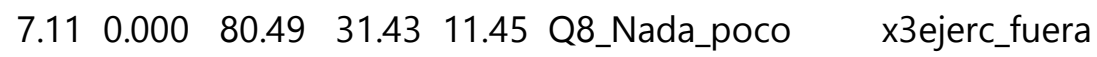


$\mathrm{m} 1 \quad 41$

$6.840 .00063 .5144 .76 \quad 20.67$ Q6_Bastante x3clases_practicas

$\mathrm{m} 2 \quad 74$

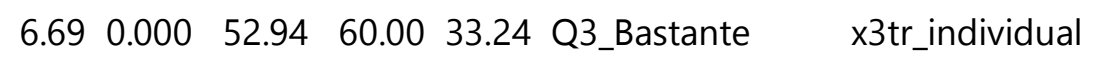

$\mathrm{m} 2 \quad 119$

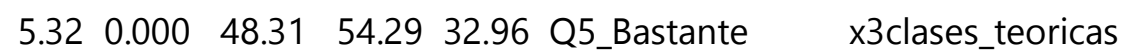

$\mathrm{m} 2 \quad 118$

$4.960 .000 \quad 43.92 \quad 61.90 \quad 41.34$ Q8_Bastante x3ejerc_fuera

$\mathrm{m} 2 \quad 148$

$\begin{array}{llllll}4.71 & 0.000 & 44.70 & 56.19 & 36.87 & \text { Q4_Bastante } \quad \text { x3tr_grupo }\end{array}$

$\mathrm{m} 2 \quad 132$

$\begin{array}{lllllll}3.78 & 0.000 & 33.33 & 94.29 & 82.96 & \text { PC } & \text { soporte_infor }\end{array}$ 297

$\begin{array}{lllll}2.70 & 0.003 & 53.33 & 15.24 & 8.38\end{array}$ Q5_Nada_poco x3clases_teoricas

$\mathrm{m} 1 \quad 30$

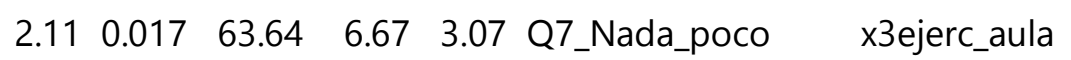

$\mathrm{m} 1 \quad 11$

$\begin{array}{lllll}-2.18 & 0.014 & 5.56 & 0.95 & 5.03\end{array}$ Smartphone soporte infor

m2 18

$\begin{array}{llllll}-2.70 & 0.003 & 11.63 & 4.76 & 12.01 & \text { Tablet } \quad \text { soporte infor }\end{array}$

$\mathrm{m} 1$ 43

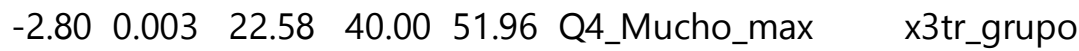

$\mathrm{m} 3 \quad 186$

$\begin{array}{llllll}-2.86 & 0.002 & 10.00 & 3.81 & 11.17 & \text { Q4_Nada_poco } \quad \text { x3tr_grupo }\end{array}$

$\mathrm{m} 1 \quad 40$

-6.65 0.00019 .8551 .4375 .98 Q6_Mucho_max x3clases_practicas

$\mathrm{m} 3 \quad 272$

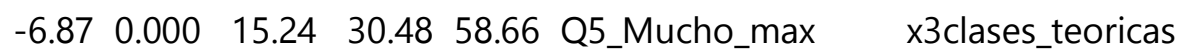

$\mathrm{m} 3 \quad 210$

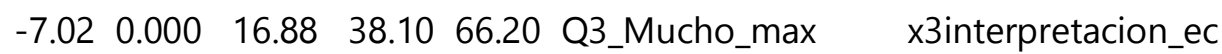

$\mathrm{m} 3 \quad 237$

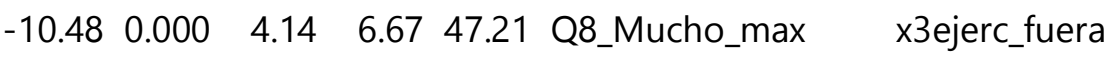

m3 169

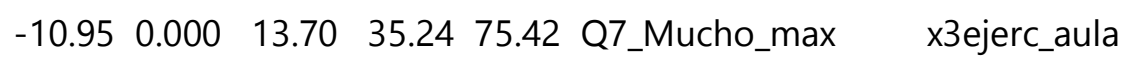
m3 270

Cluster 3 / 3

T.VALUE PROB. ---- PERCENTAGES ---- CHARACTERISTIC

IDEN WEIGHT

GRP/CAT CAT/GRP GLOBAL CATEGORIES OF VARIABLES

55.59 Cluster $3 / 3$

aa1a 199

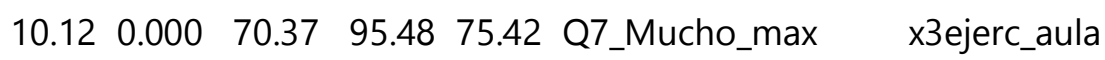
m3 270

9.610 .00069 .4994 .97 75.98 Q6_Mucho_max $\quad$ x3clases_practicas 


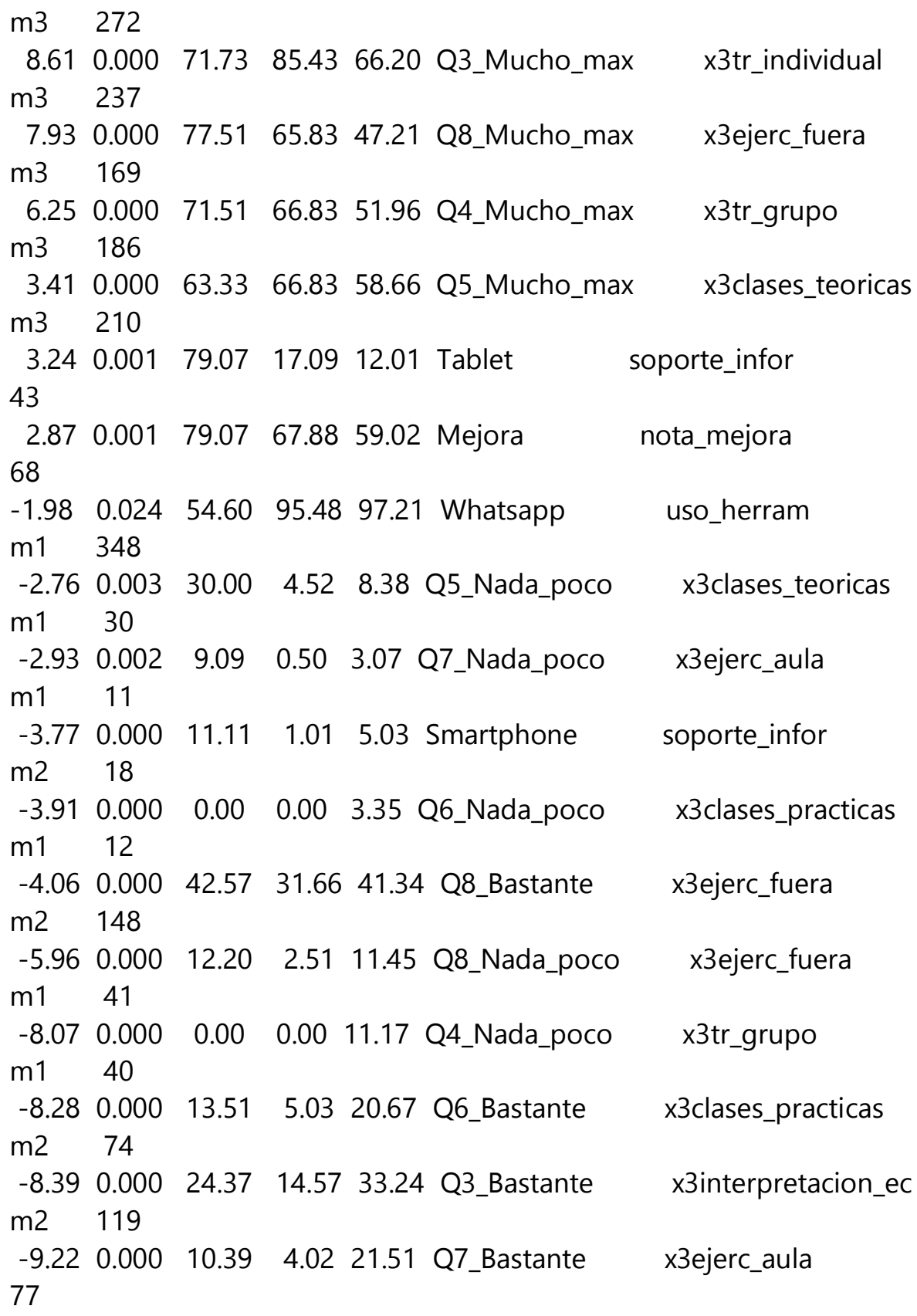

